\title{
Convex Optimization approach for Time-Optimal Path Tracking of Robots with Speed Dependent Constraints *
}

\author{
Tohid Ardeshiri, Mikael Norrlöf, Johan Löfberg, \\ Anders Hansson \\ Department of Electrical Engineering, Linköping University, SE-581 \\ 83 Sweden (e-mail: tohid.ardeshiri@isy.liu.se,mino@isy.liu.se, \\ johanl@isy.liu.se, hansson@isy.liu.se)
}

\begin{abstract}
The task of generating time optimal trajectories for a six degrees of freedom industrial robot is discussed and an existing convex optimization formulation of the problem is extended to include new types of constraints. The new constraints are speed dependent and can be motivated from physical modeling of the motors and the drive system. It is shown how the speed dependent constraints should be added in order to keep the convexity of the overall problem. A method to, conservatively, approximate the linear speed dependent constraints by a convex constraint is also proposed. A numerical example proves versatility of the extension proposed in this paper.
\end{abstract}

Keywords: Optimal Trajectory, Convex Optimization, Industrial robotics

\section{INTRODUCTION}

To maximize the productivity in modern production plants the cycle time in the robot cells is often a limiting factor, therefore time-optimal motion planning applied to robotic manipulators is of significant importance in real applications (Björkman et al., 2008). For an optimization method to be useful there are a number of requirements that have to be met. Firstly the solution, the time optimal trajectory, must be possible to compute in a short time, preferably in real time. Secondly, the optimization problem formulation must have a high degree of flexibility, which means that it must be easy to add new constraints and the constraints must be possible to parameterize in a general way. Of equal importance is that the optimization uses realistic constraints, considering both the user's demands on the tool velocity, and possibly the tool acceleration, as well as the internal robot constraints. The internal robot constraints, for example motor maximum speed, motor torque, etc. can be extracted from the specification of the motors. These constraints are further adjusted to meet thermal dissipation capacity of motor and gearbox (Leonhard, 2001). In addition when the overall lifetime of the robot is considered the constraints can also be adjusted. One of several approaches for motion planning of robotic manipulators is the so called decoupled approach (Lin et al., 1983, Shin and McKay, 1985, Shiller and Dubowsky, 1991). The decoupled approach is composed of two stages: path planning and path tracking. The path planning stage is concerned with geometric constraints and obstacle avoidance (Nyström and Norrlöf, 2003), whereas dynamic aspects such as manipulator constraints are concerns of the path tracking stage (Verscheure et al., 2009).

^ This work was supported by Vinnova's Industry Excellence Center
LINK-SIC at Linköping University, which is gratefully acknowledged.
The time-optimal path tracking stage, which is the focus of this paper, is concerned with time-optimality along a predefined path. The goal in the path planning stage is to make the robot move with as high velocity as possible without violating any constraint. To achieve this it is necessary to have a complete model of the manipulator and the actuator constraints (Shin and McKay, 1985, Bobrow et al., 1985, Shin and McKay, 1986, Pfeiffer and Johanni, 1987, Slotine and Yang, 1989, Tarkiainen and Shiller, 1993, Constantinescu and Croft, 2000). As shown in (Verscheure et al., 2009) it is straightforward to do the optimization with respect to energy instead of cycle time or a combination of both time and energy. In this paper the result in (Verscheure et al., 2009) is extended to cover speed dependent constraints, such as viscous friction in the model. In Section 2 the theory from (Verscheure et al., 2009), on which this paper is based, is briefly discussed. Section 4 covers the main contribution where it is shown how the speed dependent constraints can be added and what approximations that have to be made in order to keep the convexity of the original problem. An illustrative example is presented in Section 5. The example is based on the example from (Verscheure et al., 2009), but some additional constraints on the maximum speed of the joints are added to highlight the improved performance by using the approach suggested in this paper. Finally, Section 6 presents concluding remarks.

\section{BACKGROUND}

The motion model of an n-DOF robotic manipulator with joint angles $q \in R^{n}$ can be written as a function of the applied joint torques $\tau \in R^{n}$ and is given by the following equation (Sciavicco and Siciliano, 2000),

$$
\tau=M(q) \ddot{q}+C(q, \dot{q}) \dot{q}+F_{s}(q, \dot{q}) \operatorname{sgn}(\dot{q})+G(q) .
$$


If the path is given as a function, $q(s)$, in the joint space, where $s$ is an index function that parameterize the path, then the path dynamics is captured by the scalar function $s(t)$ with constraint, $\dot{s}(t) \geq 0$. The trajectory $q(t)$ and its derivatives can now be expressed as,

$$
\begin{aligned}
& \dot{q}(s(t))=q^{\prime}(s(t)) \dot{s}(t), \\
& \ddot{q}(s(t))=q^{\prime}(s(t)) \ddot{s}(t)+q^{\prime \prime}(s(t)) \dot{s}^{2}(t),
\end{aligned}
$$

which is also exploited in for example (Shin and McKay, 1985), (Bobrow et al., 1985), and (Pfeiffer and Johanni, 1987). The key to solve the minimal time trajectory for the robotics system, given the path geometry, is to use (2) in (1) and parameterize $s(t)$ so that the acceleration is the free variable. If dynamic friction is neglected, the time-optimal path tracking problem can be formulated as in (Verscheure et al., 2009), where the original problem is posed as a convex optimization problem,

$$
\begin{aligned}
\underset{a(.), b(.), \tau(.)}{\operatorname{minimize}} & \int_{0}^{1} \frac{1}{\sqrt{b(s)}} d s \\
\text { s.t. } \tau(s) & =m(s) a(s)+c(s) b(s)+g(s) \\
b(0) & ={\dot{s_{0}}}^{2} \\
b(1) & ={\dot{s_{T}}}^{2} \\
b^{\prime}(s) & =2 a(s) \\
b(s) & \geq 0 \\
b(s) & \leq \bar{b}(s) \\
\underline{\tau}(s) & \leq \tau(s) \leq \bar{\tau}(s) \\
\underline{f}(s) & \leq f(s) a(s)+h(s) b(s) \leq \bar{f}(s)
\end{aligned}
$$

for $s \in[0,1]$. With this problem formulation $a(s)$ and $b(s)$ represent the acceleration and the square of the speed along the path coordinate,

$$
\begin{aligned}
b(s) & =\dot{s}^{2}, \\
a(s) & =\ddot{s} .
\end{aligned}
$$

It is easy to impose Cartesian speed and acceleration constraints if the path length $l(s), \dot{l}(s) \geq 0$, is introduced. It is also shown in (Verscheure et al., 2009) how the discretized problem can be posed as a second order cone program, SOCP, which can be solved very efficiently with solvers, such as SeDuMi (Sturm, 1999), SDPT3 (Toh et al., 1999).

\section{ROBOT AND CONSTRAINTS MODELING}

In a real robot application where the goal is to fully utilize the robot performance the constraints used in the optimization problem formulation in (3) to (11) can be deficient. In general there are two limitations that has to be considered in the drive system (Beaty and James L. Kirtley, 1998). The first one is that the armature current is limited due to the heat produced in the motor, a thermal requirement. The second one is that the DCvoltage that can be used to drive the motors is bounded. This is especially evident at high speeds where the counterelectromotive force (counter-EMF) will impose a constraint on the torque and top speed for the motors. The counter-EMF is proportional to the angular velocity of the motor, and it will impose an approximately linear decrease of the available torque as a function of angular velocity. A typical torque versus speed capability specification for a brushless DC-motor is shown in Fig. 1. The constant

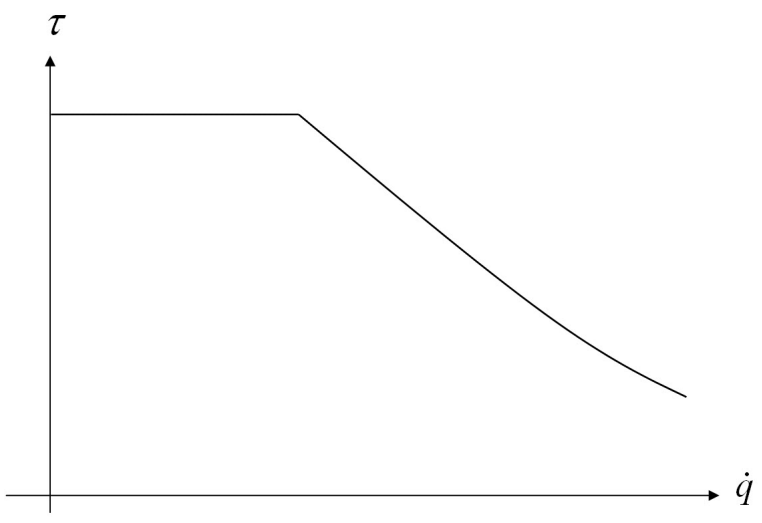

Fig. 1. Typical torque versus speed characteristics for an electrical brushless DC-motor.
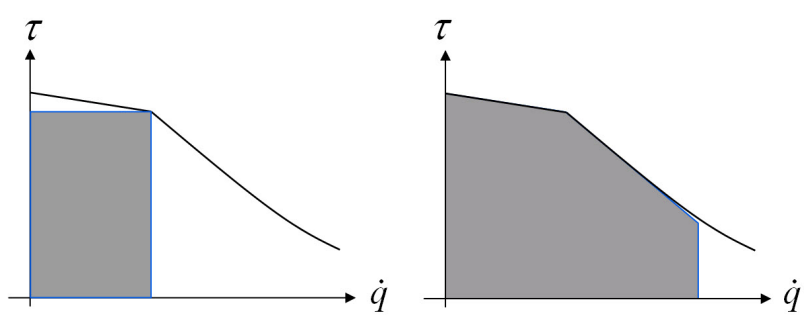

Fig. 2. Resulting constraints when considering constant torque constraint as in Equation (10) (left), and speed dependent constraint (right).

torque at low speeds comes from the thermal dissipation requirement which limits the maximum current in the motor. At a certain speed the torque is reduced due to a limited DC-bus voltage.

As indicated in the conclusion of (Verscheure et al., 2009), the dynamic friction torque can also be significant part of the total torque, and with the assumption that the available torque is constant this torque is neglected, or must be handled by a conservative torque constraint. In Fig. 2 the resulting constraints are shown when friction and the limited DC-level is included in the specification of the available torque. If a constant torque constraint is used the torque and maximum speed must be chosen so that the result is conservative, as indicated in the left diagram in Fig. 2. The speed constraint,

$$
\underline{\dot{q}} \leq q^{\prime}(s) \dot{s} \leq \overline{\dot{q}}
$$

can be transformed into (9) by some simple manipulations. When a speed dependent torque constraint can be used the torque can be utilized in a much more efficient way, as in shown in the right diagram in Fig. 2. The friction torque limits the available torque in acceleration, while in a situation where a joint is decelerating, the available torque is increased by friction, hence making it possible to have a higher deceleration performance compared to acceleration performance. In the examples in this paper it is assumed that the constraint is symmetric in acceleration and deceleration, but it is straight forward to extend to a non-symmetric constraint. In the next section it is explained how it is possible to introduce the new speed dependent constraints in the convex optimization problem. 


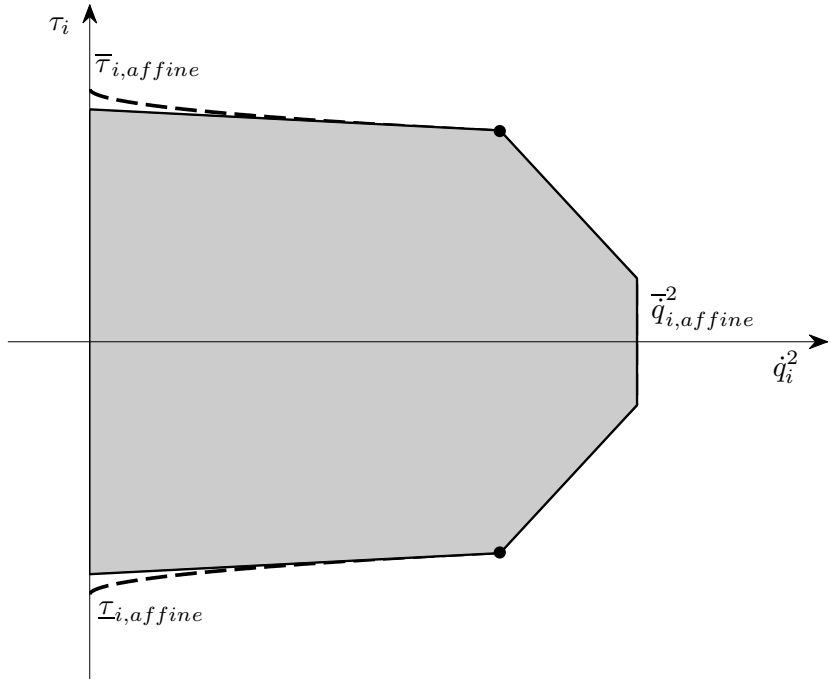

Fig. 3. The non-convex true feasible set derived from the right diagram in Fig. 2 is approximated by a set of affine constraints. The true actuator's constraints is represented by the dashed line. The approximation of the feasible set by a convex set is illustrated by the hatched area.

\section{CONVEX SPEED DEPENDENT CONSTRAINTS}

From Section 3 it can be concluded that, from a robotic application point of view, it is important to extend the solution in (Verscheure et al., 2009) to include speed dependent constraints. A typical model of maximum torque of an actuator is shown in Fig. 2 where the available torque is modeled by a set of affine constraints in the torquejoint velocity space. Addition of the speed dependent constraints in Fig. 2 to the time optimal path tracking problem, does however not preserve the convex formulation of the optimization problem. It can be shown that constraints that are affine in joint velocity are no longer convex in the parameterization where the joint speed square is used. Consider an affine constraint as

$$
\tilde{T} \tau_{i}(s)+W_{i} \dot{q}_{i}(s) \leq P_{i 1} .
$$

Using (2a) this equation can be rewritten as,

$$
\begin{aligned}
& \tilde{T} \tau_{i}(s)+W_{i} q_{i}^{\prime}(s) \dot{s} \leq P_{i 1}, \\
& \tilde{T} \tau_{i}(s)+W_{i} q_{i}^{\prime}(s) \sqrt{b(s)} \leq P_{i 1} .
\end{aligned}
$$

The feasible set described by (15b) is not convex with respect to $b(s)$ for some values of $q_{i}^{\prime}(s)$. To be able to guarantee a convex constraint, the true feasible set with respect to $\dot{q}_{i}$ and $\tau_{i}$ is approximated by a set which is convex with respect to $\dot{q}_{i}^{2}$ and $\tau_{i}$ and which remains convex under the nonlinear transformation in (12a). In Fig. 3 the approximated constraint is illustrated for the constraint in Fig. 2.

Now we show that with the modified constraint we will preserve the convex formulation of the time optimal path tracking problem with respect to $a, b$ and $\tau$.

From (12a) we can write

$$
\left(\begin{array}{c}
b(s) \\
\tau_{i}
\end{array}\right)=F\left(\dot{q}_{i}^{2}(s), \tau_{i}\right)=\left(\begin{array}{crc}
q_{i}^{\prime-2}(s) & 0 \\
& 0 & 1
\end{array}\right)\left(\begin{array}{c}
\dot{q}_{i}^{2}(s) \\
\tau_{i}
\end{array}\right)
$$

where $F(\cdot, \cdot)$ is an affine function. For any convex set $S \subset$ $R^{2}$ it holds that $F(S)$ is convex (Boyd and Vandenberghe, 2004 ), and hence this is especially true for the convex set in Fig. 3.

Let $(17)$ be an affine constraint with respect to $\tau_{i}$ and $\dot{q}_{i}^{2}$.

$$
T_{i 1} \tau_{i}(s)+\bar{U}_{i 1} \dot{q}_{i}^{2}(s) \leq P_{i 1}
$$

It can be rewritten as

$$
T_{i 1} \tau_{i}(s)+U_{i 1}(s) b(s) \leq P_{i 1}
$$

where

$$
U_{i 1}(s)=\bar{U}_{i 1} q_{i}^{\prime 2}(s)
$$

As a result the approximation of the feasible set with respect to $\dot{q}_{i}^{2}$ and $\tau_{i}$ results in a set of affine inequality constraints with respect to the optimization variables $b(s)$ and $\tau_{i}(s)$.

The $j$ th affine constraints for the actuator $i$ and after discretization results in

$$
T_{i j} \tau_{i}^{k}+U_{i j}\left(s^{k+1 / 2}\right) b^{k+1 / 2} \leq P_{i j}
$$

where

$$
U_{i j}\left(s^{k+1 / 2}\right)=\bar{U}_{i j} q_{i}^{\prime 2}\left(s^{k+1 / 2}\right)
$$

and where

$$
\begin{aligned}
& s^{k+1 / 2}=\left(s^{k}+s^{k+1}\right) / 2 \\
& b^{k+1 / 2}=\left(b^{k}+b^{k+1}\right) / 2
\end{aligned}
$$

This can be summarized as,

$$
\left(\begin{array}{cccc}
T_{11} & 0 & \ldots & 0 \\
T_{12} & 0 & \ldots & 0 \\
\ldots & 0 & \ldots & 0 \\
T_{1 m_{1}} & 0 & \ldots & 0 \\
0 & T_{21} & \ldots & 0 \\
0 & T_{22} & \ldots & 0 \\
0 & \ldots & \ldots & 0 \\
0 & T_{2 m_{2}} & \ldots & 0 \\
\ldots & \ldots & \ldots & \ldots \\
0 & 0 & \ldots & T_{n m_{n}}
\end{array}\right)\left(\begin{array}{c}
\tau_{1}^{k} \\
\tau_{2}^{k} \\
\ldots \\
\tau_{n}^{k}
\end{array}\right)+\left(\begin{array}{c}
U_{11 / 2}^{k+1 / 2} \\
U_{12}^{k+1 / 2} \\
\ldots \\
U_{1 m_{1}}^{k+1 / 2} \\
U_{21 / 2}^{k+1} \\
U_{22}^{k+1 / 2} \\
\ldots \\
U_{2 m_{2}}^{k+1 / 2} \\
\ldots \\
U_{n m_{n}}^{k+1 / 2}
\end{array}\right)
$$

or in matrix form as

$$
T \tau^{k}+U^{k+1 / 2} b^{k+1 / 2} \leq P
$$

If we add the new constraint in Equation (24) to the continuous time-optimal path tracking problem it results in 


$$
\begin{aligned}
\underset{a(.), b(.), \tau(.)}{\operatorname{minimize}} & \int_{0}^{1} \frac{1}{\sqrt{b(s)}} d s \\
\tau(s) & =m(s) a(s)+c(s) b(s)+g(s) \\
b(0) & ={\dot{s_{0}}}^{2} \\
b(1) & =\dot{s}_{T}^{2} \\
b^{\prime}(s) & =2 a(s) \\
b(s) & \geq 0 \\
\underline{f}(s) & \leq f(s) a(s)+h(s) b(s) \leq \bar{f}(s) \\
T \tau(s) & +U(s) b(s) \leq P
\end{aligned}
$$

for $s \in[0,1]$.

In the discretized representation of the problem as an SOCP we get,

$$
\begin{aligned}
& \underset{a^{k}, b^{k}, \tau^{k}, d^{k}, c^{k}}{\operatorname{minimixe}} \sum_{k=0}^{K-1} 2 \Delta s^{k} d^{k} \\
& \tau^{k}=m\left(s^{k+1 / 2}\right) a^{k}+c\left(s^{k+1 / 2}\right) b^{k+1 / 2}+g\left(s^{k+1 / 2}\right) \\
& b^{0}={\dot{s_{0}}}^{2} \\
& b^{K}={\dot{s_{T}}}^{2} \\
& b^{k+1}-b^{k}=2 a^{k} \Delta s^{k} \\
& b^{k} \geq 0 \text { and } b^{K} \geq 0 \\
& f\left(s^{k+1 / 2}\right) \leq f\left(s^{k+1 / 2}\right) a^{k}+h\left(s^{k+1 / 2}\right) b^{k+1 / 2} \\
& f\left(s^{k+1 / 2}\right) a^{k}+h\left(s^{k+1 / 2}\right) b^{k+1 / 2} \leq \bar{f}\left(s^{k+1 / 2}\right) \\
& \left\|c^{k}+c^{2+1}-d^{k}\right\| \leq c^{k}+c^{k+1}+d^{k} \\
& \left\|\begin{array}{c}
2 c^{k} \\
b^{k}-1
\end{array}\right\| \leq b^{k}+1 \\
& T \tau^{k}+U^{k+1 / 2} b^{k+1 / 2} \leq P^{k+1 / 2}
\end{aligned}
$$

for $k=0,1, \ldots, K-1$.

Thus far all actuators are assumed to be rotational actuators, but all the results are trivially valid for translational joints with force and linear speed instead of torque and angular speed. In (Verscheure et al., 2009) the time-optimal path tracking problem can handle scalarized multi-objective optimization problem. This general problem formulation enables trading off time-optimality with dissipated thermal energy and the integral of the rate of change of the torque. It should be noted that introduction of this new type of constraint is not in conflict with the general formulation of the path tracking problem.

\section{NUMERICAL EXAMPLE}

The MATLAB implementation is based on the example used in (Verscheure et al., 2009) with some modifications that will be motivated in more detail below. The first observation that we can make from the example is that no constraints are active for joints 4-6 and therefore the results for these joints will not be presented here. The second observation from the example in (Verscheure et al., 2009 ) is that at high joint speeds the maximum torque is not utilized. In the example that will be shown here the speed constraints were set at $90 \%$ of the maximum attained velocity from the example in (Verscheure et al., 2009). The optimization solution is found using YALMIP (Löfberg, 2004) and SeDuMi (Sturm, 1999).
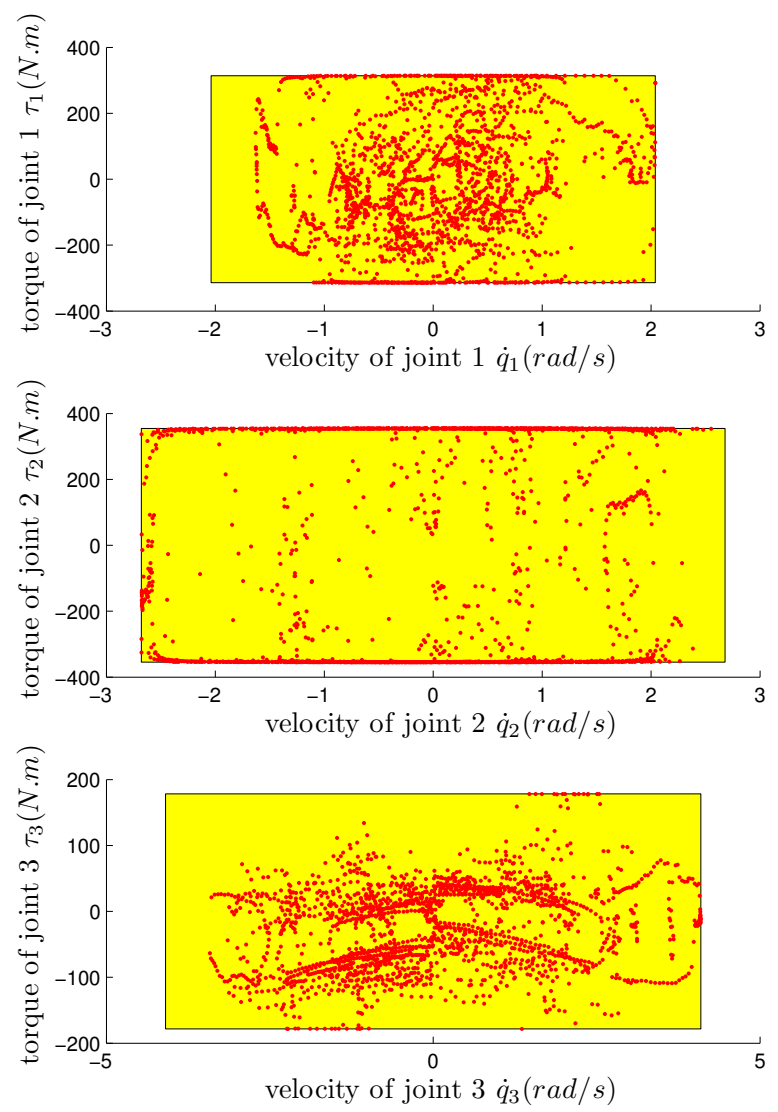

Scenario 1: optimal torque versus optimal velocity Scenario 1: feasible set w.r.t. joint's torque and velocity

Fig. 4. Scenario 1: Optimal torque and angular velocity calculated by the algorithm with box constraints.

To compare the proposed approach with speed dependent torque constraints with the box constraints approach the result from two scenarios are compared.

- Scenario 1: In this scenario rotational speed constraints are imposed on joints $1-3$. The maximum speed constraint is set at $90 \%$ of maximum absolute velocity for each of the first three joints in the example presented in (Verscheure et al., 2009). The calculated optimal joint torque versus its angular speed for this scenario is plotted in Fig. 4.

- Scenario 2: In this scenario an affine set of constraints is imposed on each one of the first three joints. This set of constraint is approximated by another affine set of constraint which is convex with respect to $\tau_{i}$ and $\dot{q}_{i}^{2}$. The first set of affine constraints is illustrated in the right diagram in Fig. 2 and the approximation is illustrated in Fig. 3. Fig. 5 shows the resulting optimal joint torque versus joint speed square.

To motivate the choice of the constraints shown in Fig. 2 and Fig. 6, it is the constraint set given by Scenario 2 that has to be the starting point. This set is based on the physical constraints on the motor and the drive system, considering viscous friction and the counter EMF. Additional constraints can be added if life time considerations 

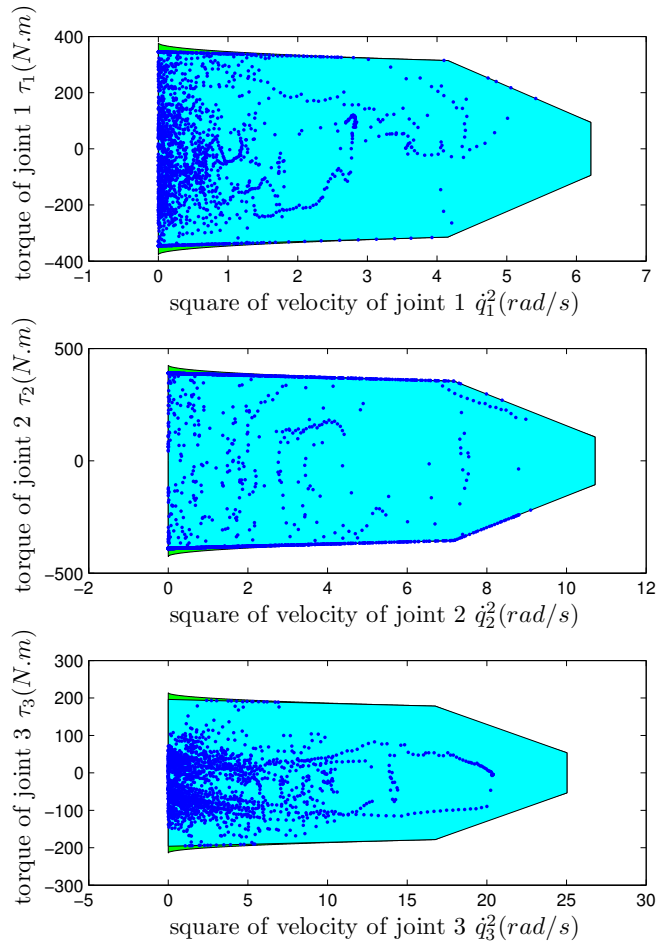

feasible set of scenario 2 before approximation

feasible set of scenario 2 after approximation with affine functions Scenario 2: optimal torque vs square of optimal velocity

Fig. 5. Optimal torque versus the square of optimal velocity of each joint is plotted. Activation of constraints are illustrated.

are included as well but these additional constraints are not considered in this example. To obtain a box constraint it is necessary to match the constraint to the physical constraint and fit the box constraint inside the physical model based constraint, as shown in the left diagram in Fig. 2. In Fig. 6 the constraints in Scenarios 1 and 2 are shown. In this case it is decided to get combine highest possible speed and torque in Scenario 1. By decreasing the top speed a higher torque could be used at low speeds, for example if the robot is used in contact applications, such as drilling and deburring. A higher top speed could be achieved if the maximum torque is decreased. From comparison of constraints depicted in Fig. 2 it can be perceived that the maximum torque of the actuator and its maximum speed in the affine constraint set are higher than their counterpart in the constant torque constraint.

The calculated optimal torque for the second scenario is shown in Fig. 5. It can be seen that the algorithm utilizes the extra available torque and speed for the three actuators. Although some of the extra torque cannot be utilized due to the convex approximation which leads to cut-off of the non-convex part of the feasible set, the utilized extra torque and speed has lead to a reduced cycle time. The cycle time for the given trajectory is decreased from $9.83 \mathrm{~s}$ to $9.38 \mathrm{~s}$, which means a cycle time reduction of $4.6 \%$. The scatter of calculated optimal torque versus optimal speed at each segment of the trajectory for both scenarios and their feasible set are depicted in Fig. 6. It should be noted that the speed dependent constraint which was introduced in this article is a versatile tool to
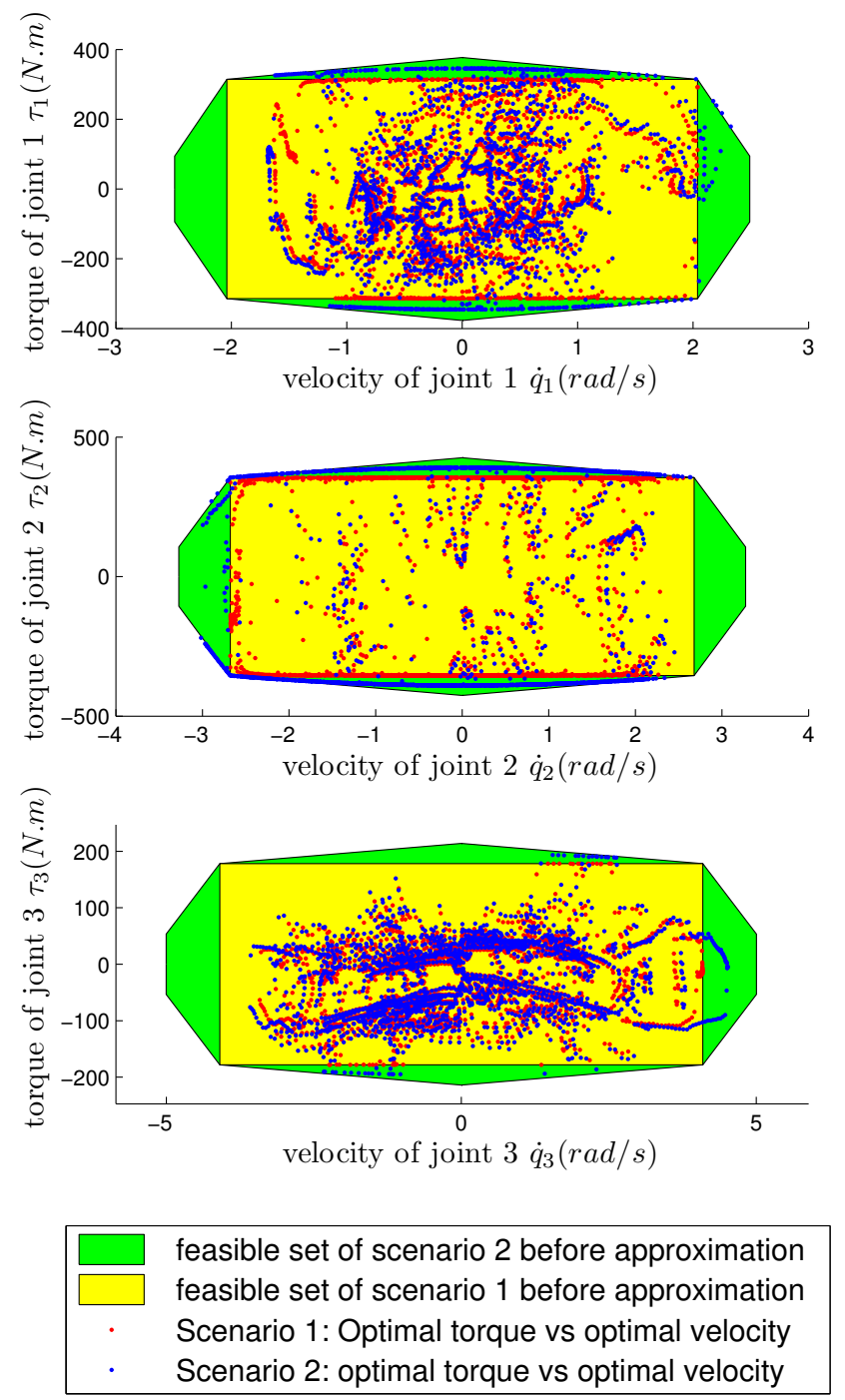

Fig. 6. Scatter of calculated optimal torque and optimal velocity for both scenarios overlaid.

capture the true constraints of the actuator and utilize the most of the available torque and speed from the actuator without violating the constraints. The constraints should be convex with respect to $\tau_{i}$ and $\dot{q}_{i}^{2}$ to preserve the convex formulation of the problem.

\section{CONCLUSION}

The introduction of speed dependent constraints to the convex optimization formulation of the time optimal path tracking problem has shown encouraging results. By utilization of more torque at lower speeds and more speed at lower torques the cycle time for an example trajectory is reduced by almost $5 \%$. We have used a slightly modified example from (Verscheure et al., 2009) to illustrate the result, but it could be noted that the cycle time reduction could be significantly higher in other examples. The top speed is increased $20 \%$ in Scenario 2 compared to Scenario 1 , and in a trajectory where the maximum speed constraint is active the cycle difference can be close to $20 \%$. It is shown in the paper how friction and motor specification can be included in the constraints. The modeling guideline 
can be used to capture details of true feasible set while the convexity of the problem is preserved.

\section{REFERENCES}

Beaty, H.W. and James L. Kirtley, J. (eds.) (1998). Electric Motor Handbook. McGraw-Hill Handbooks. McGraw-Hill Book Company.

Björkman, M., Brogardh, T., Hanssen, S., Lindström, S.E., Moberg, S., and Norrlöf, M. (2008). A new concept for motion control of industrial robots. In Proc. 17th IFAC World Congress.

Bobrow, J.E., Dubowsky, S., and Gibson, J.S. (1985). Time-optimal control of robotic manipulators along specified paths. Int. J. Robot. Res., 4(3), 3-17.

Boyd, S. and Vandenberghe, L. (2004). Convex Optimization. Cambridge Univ. Press [online] Available. URL http://www.ee.ucla.edu/ vandenbe/cvxbook.html.

Constantinescu, D. and Croft, E.A. (2000). Smooth and time-optimal trajectory planning for industrial manipulators along specified paths. J. Robot. Syst., 17(5), 233-249.

Leonhard, W. (2001). Control of Electrical Drives. Springer-Verlag, third edition.

Lin, C.S., Chang, P.R., and Luh, J.Y.S. (1983). Formulation and optimization of cubic polynomial joint trajectories for industrial robots. IEEE Trans. Autom. Control, 28(12), 1066-1074.

Löfberg, J. (2004). Yalmip : A toolbox for modeling and optimization in MATLAB. In Proceedings of the CACSD Conference. Taipei, Taiwan. URL http://users.isy.liu.se/johanl/yalmip.

Nyström, M. and Norrlöf, M. (2003). Path generation for industrial robots. In Mekatronikmöte 2003. Gteborg, Sweden. URL http://www. control.isy.liu.se/publications/.

Pfeiffer, F. and Johanni, R. (1987). A concept for manipulator trajectory planning. IEEE J. Robot. Autom., 3(2), $115-123$

Sciavicco, L. and Siciliano, B. (2000). Modelling and Control of Robot Manipulators. Springer.

Shiller, Z. and Dubowsky, S. (1991). On computing the global time-optimal motions of robotic manipulators in the presence of obstacles. IEEE Trans. Robot. Autom., $7(6), 786-797$.

Shin, K.G. and McKay, N.D. (1985). Minimum-time control of robotic manipulators with geometric path constraints. IEEE Trans. Autom. Control, 30(6), 531541.

Shin, K.G. and McKay, N.D. (1986). A dynamic programming approach to trajectory planning of robotic manipulators. IEEE Trans. Autom. Control, 31(6), 491500.

Slotine, J.J.E. and Yang, H.S. (1989). Improving the efficiency of timeoptimal path-following algorithms. IEEE Trans. Robot. Autom., 5(1), 118-124.

Sturm, J.F. (1999). Using SeDuMi 1.02, a MATLAB toolbox for optimization over symmetric cones. $O p$ timization Methods and Software, 11-12(1-4), 625-653. Available from http://sedumi.ie.lehigh.edu.

Tarkiainen, M. and Shiller, Z. (1993). Time optimal motions of manipulators with actuator dynamics. In Proc. IEEE Int. Conf. Robot. Autom., volume 2, 725730. Atlanta, GA.
Toh, K.C., Todd, M.J., and Tütüncü, R.H. (1999). SDPT3 - a Matlab software package for semidefinite programming, version 2.1. Optimization Methods and Software, 11-12(1-4), 545-581. Available from http: //www.math.nus.edu.sg/ mattohkc.

Verscheure, D., Demeulenaere, B., Swevers, J., De Schutter, J., and Diehl, M. (2009). Time-optimal path tracking for robots: A convex optimization approach. $A u$ tomatic Control, IEEE Transactions on, 54(10), 2318 -2327 . 